\title{
ATENCIÓN ODONTOLÓGICA PREVENTIVA Y RESTAURATIVA EN PACIENTES NIÑOS DE LAS PARROQUIAS DEL CANTÓN CUENCA POR EL PROYECTO DE VINCULACIÓN. PERIOdO 2019-2020.
}

\author{
Preventive and restorative dental care in children patients of the parishes of the \\ Cuenca canton for the bonding project. 2019-2020 period.
}

\begin{abstract}
Alisson Virginia Machado Quinde ${ }^{1}$, Mayra Casandra Samaniego Urrego ${ }^{1}$, Kelly Gabriela Zaldúa Salazar $^{1}$, Sandra Patricia Saquisili Suquitana ${ }^{2}$.

1 Alumna, de la Carrera de Odontología, rotante del programa de investigación, Universidad Católica de Cuenca, Cuenca, Ecuador.

${ }^{2}$ Docente/ investigador, Carrera de Odontología, Universidad Católica de Cuenca, Cirujano Maxilofacial, Cuenca, Ecuador.
\end{abstract}

\section{Resumen}

Objetivos: Determinar la frecuencia de la atención odontológica preventiva y restaurativa en pacientes niños de las parroquias del cantón Cuenca, periodo 2019-2020. Materiales y métodos: El presente estudio fue de tipo documental, diseño transversal retrospectivo descriptivo. La muestra consistió en un total de fichas de 219 niños anónimos atendidos bajo el proyecto de vinculación en Cuenca en el periodo 2019-2020. Se realizó estadística descriptiva para determinar la frecuencia de la atención odontológica mediante el programa Excel 2016. Resultados: La mayor frecuencia de actividades odontológicas preventivas fueron los sellantes con un $22 \%$, en las actividades de restauración fue la operatoria dental con un $14 \%$, no hubo diferencias significativas entre sexo y los niños más atendidos fueron en edades de 0 a 3 años con un $53 \%$. Conclusiones: Se observa la necesidad de implementar más programas de intervención con el objetivo de comunicar a la comunidad la importancia de transmitir hábitos de higiene oral desde la edad temprana.

Palabras clave: caries dentales, escolaridad, comunidad dentista.

\begin{abstract}
Aim: To determine the frequency of preventive and restorative dental care in child patients from the parishes of the Cuenca canton, period 2019-2020. Material and methods: This study was documentary, with a descriptive retrospective crosssectional design. The sample consists of a total of 219 anonymous children served under the bonding project in Cuenca in the period 2019-2020. Descriptive statistics were performed to determine the frequency of dental care. Results: The highest frequency of preventive dental activities were the sealants with $22 \%$, in the restoration activities it was the dental operation with 14\%, there were no differences in sex and the most attended children were aged 0 to 3 years. with 53\%. Conclusions: There is a need to implement more intervention programs in order to communicate to families the importance of transmitting good oral hygiene habits from an early age.
\end{abstract}

Key words: dental caries, schooling, community dentistry.

\section{Introducción}

En la actualidad la salud bucal es fundamental para que el ser humano goce de una buena calidad de vida. Según la Organización Mundial de la salud (OMS) define la salud bucal como la ausencia de dolor orofacial, cáncer de boca o de garganta, entre otros trastornos que limitan la habilidad de morder, masticar, sonreír y hablar, al mismo tiempo que conllevan consecuencias negativas en el bienestar psicosocial. $^{1}$

Las principales patologías orales incluyen: caries, gingivitis, las periodontopatías, placa bacteriana y la 
fluorosis dental de las cuales la más prevalente es la caries., ${ }^{2,3}$ La caries dental se da con mayor frecuencia principalmente en la infancia, con una prevalencia entre el $60 \%$ y $90 \%$ de los escolares en todo el mundo. El factor más relevante con respecto a la incidencia de caries en los escolares es el excesivo consumo de azúcares en la dieta, lo que provoca un ambiente bucal ácido favorable para la colonización de bacterias cariogénicas. La caries inicia en el esmalte como una mancha blanca y continua con un avance dinámico hasta llegar a una cavitación en la pieza afectada. Se ha demostrado que la condición del nivel socioeconómico juega un rol importante en la prevalencia del desarrollo de caries. ${ }^{4}$

La OMS imparte la educación para la salud, con la finalidad de promover procesos educativos en las instituciones y en los centros de salud de la comunidad que permita al individuo conocer sobre la enfermedad y sus factores de riesgo, haciéndolos responsables por su propia salud. La atención odontológica es esencial desde su nacimiento para evitar enfermedades bucales. ${ }^{4}$ Además, como prevención de caries dental los niños y los padres deben estar informados sobre la correcta higiene oral para educar tempranamente a sus hijos en la obtención de conductas que permitan buenos hábitos en la salud bucal., 5,6 así como también incluirlos en actividades y programas que ofrecen las escuelas y la comunidad para la promoción de la salud y prevención de enfermedades.

En Ecuador la institución encargada de cuidar por la salud de la población es el Ministerio de Salud Pública, creada desde el 16 de junio de 1967, el mismo desarrolló un Plan Nacional de Salud Bucal que garantiza el derecho a la atención en la salud bucodental de la población. Dentro del nuevo modelo de atención integral de salud, intercultural familiar y comunitaria, el Plan Nacional de Salud Bucal está orientado principalmente en estrategias de promoción y prevención de la enfermedad. $^{7}$

La salud es un derecho que el estado debe proporcionar a las diferentes comunidades, por lo tanto, el gobierno ha dispuesto un servicio de salud universal y gratuita, según sea el análisis epidemiológico sobre las principales enfermedades que afectan a los individuos, teniendo en cuenta factores como género, etnia, edad, clases sociales, educación e ingresos que generan. La atención estomatológica es importante para mantener un estado de bienestar de los individuos. Por lo tanto, la asistencia es obligatoria para los estudiantes egresados de Odontología que indica el Plan Nacional de Salud Rural, dirigidos a brindar atención odontológica en comunidades con un nivel socioeconómico bajo. El Plan de Desarrollo Integral de Odontología tiene como objetivo implementar programas educativos de prevención y de higiene en comunidades rurales.
Para el desarrollo del servicio de salud, el gobierno ha modificado las infraestructuras de subcentros de salud, ha brindado insumos odontológicos según la necesidad de la población y ha implementado equipos tecnológicos que rinden una mejor condición de trabajo para los profesionales del área de Estomatología y así poder brindar un servicio de salud de calidad para las comunidades. ${ }^{7}$

Los métodos preventivos de enfermedades buco dentales que ofrecen los servicios de salud son la indicación de una dieta balanceada, la realización de tratamientos preventivos como son el uso de sellantes durante el cambio de dentición decidua a permanente, la aplicación de flúor tópico adhesivo y el tratamiento operatorio de las caries activas. $^{3}$ Por otro lado se destaca la importancia de la motivación y la educación para mantener la salud oral en óptimas condiciones, con la ayuda de herramientas básicas como técnicas de higiene, el uso de seda dental, los dentífricos con flúor y los enjuagues bucales antibacterianos. ${ }^{2}$

La salud oral ha sido afectada por conocimientos no profesionales basados en costumbres y culturas resultado de la poca educación y la condiciónes ambientales, esto ha variado a lo largo de la vida. ${ }^{2,3}$ El objetivo del presente estudio fue determinar la frecuencia de la atención odontológica preventiva y restaurativa en pacientes niños de las parroquias del cantón Cuenca por programas de vinculación, periodo 2019-2020.

\section{Materiales y métodos}

Se realizó un estudio de tipo documental, de diseño transversal retrospectivo descriptivo. Para determinar el estudio se analizó "El informe de actividades" que nos proporcionó el departamento de vinculación con la sociedad de la carrera de odontología de la Universidad Católica de Cuenca durante los meses de octubre 2019 a enero 2020. Los datos informativos constaron de un total de fichas de 219 niños anónimos que fueron atendidos bajo el proyecto de vinculación: "ALTERACIONES BIOLÓGICAS Y PSICOLÓGICAS EN MENORES DE 6 AÑOS DE LAS PARROQUIAS TURI, SININCAY, RICAURTE, EL VALLE Y BAÑOS DEL CANTÓN CUENCA 2019 - 2020." En las escuelas Alfonso Carrión, Joel Monroy, Enriqueta Cordero Dávila y Pitufos del Valle en las diferentes parroquias del cantón Cuenca.

El informe nos proporcionó datos importantes como la edad, el sexo, el lugar ,nombre de las unidades educativas y las actividades odontológicas realizadas que incluyeron charlas educacionales, fluorizaciones, profilaxis, aplicación de sellantes, operatorias y exodoncias. 
El total de la muestra constó de 219 niños. Se incluyeron los datos de los niños de un rango de edad entre 0 a 15 años y los que presentaron consentimientos y asentimientos informados por el representante legal. Se excluyeron los datos de los niños que no presentaron consentimientos informados y fichas con errores.

Se realizó estadística descriptiva, utilizando la frecuencia y el porcentaje para las variables representadas en tablas de Excel. Según la atención odontológica, el grupo etario, el sexo y el lugar donde fueron atendidos según los datos informativos.

Esta investigación no involucra conflictos éticos porque su estudio se realizó sobre una base de datos.

\section{Resultados}

Del total de la población examinada que fueron 219 niños, el sexo masculino tuvo mayor atención odontológica con un $51 \%$ mientras que el sexo femenino con un $49 \%$.

\begin{tabular}{lcc}
\hline Rango de Edad & Número de Pacientes & $\%$ \\
\hline $\mathbf{0 - 3}$ & 115 & 53 \\
$\mathbf{4 - 7}$ & 55 & 25 \\
$\mathbf{8 - 1 1}$ & 30 & 14 \\
$\mathbf{1 2 - 1 5}$ & 19 & 8 \\
Total & 219 & 100 \\
\hline
\end{tabular}

Tabla 1. Total de pacientes atendidos según el rango de edad.

En la tabla 1 podemos observar con respecto a la edad que el grupo etario de 0-3 años corresponde al grupo que tuvo más atención odontológica con un $53 \%$ y que pertenecen al Centro de Educación Básica Pitufos del Valle.

\begin{tabular}{lcc}
\hline \multicolumn{1}{c}{ Actividades de prevención } & Frecuencia & \% \\
\hline Charla de prevención & 195 & 17 \\
Diagnóstico & 195 & 17 \\
Profilaxis & 210 & 18 \\
Fluorizaciones & 120 & 10 \\
Sellantes & 253 & 22 \\
Total & 973 & 85 \\
\hline Actividades de restauración & & \\
Operatorias & 163 & 14 \\
Exodoncia & 7 & 1 \\
Total & 170 & 15 \\
\hline
\end{tabular}

Tabla 2. Frecuencia de las actividades realizadas.

En la tabla 2 podemos observar que las actividades que más se realizaron fueron las de prevención con un porcentaje de $85 \%$, seguida de las actividades de restauración con un porcentaje de $15 \%$.

\begin{tabular}{lccc}
\hline Unidades Educativas & Número de Pacientes & $\%$ & Grado de escolaridad \\
\hline Pitufos Del Valle & 95 & 43 & Inicial básica \\
Alfonso Carrión & 70 & 32 & Primaria y secundaria \\
Joel Monroy & 34 & 16 & Primaria y secundaria \\
& 20 & 9 & Primaria y secundaria \\
Enriqueta Cordero Dávila & 219 & 100 & \\
Total & & & \\
\hline
\end{tabular}

Tabla 3. Número de pacientes atendidos según la escuela.

En la tabla 3 podemos observar la cantidad de niños atendidos según la escuela se obtuvo que el centro de Educación Básica Pitufos Del Valle conto con un mayor número de participantes durante la atención odontológica con un $43 \%$.

\section{Discusión}

En el presente estudio destacamos una diferencia mínima de atención odontológica de acuerdo al sexo demostrando una mayor frecuencia en los niños con un porcentaje del $51 \%$ del total de la población. Sin embargo, en el estudio de Mejía y col. determinaron que los pacientes más atendidos fueron del sexo femenino con un porcentaje del $57 \% .^{8}$ Se estima que la caries dental afecta a los niños de ambos sexos sin diferencias significativas. Sin embargo, es indispensable la atención odontológica preventiva y restaurativa en niños con alto riesgo de caries con la finalidad de disminuir la prevalencia o la progresión de la enfermedad.

En cuanto a la edad, nuestro estudió determinó que los niños más atendidos tanto en métodos preventivos como restaurativos fueron en edades de 0 a 3 años con un porcentaje del $53 \%$, datos que concuerdan de forma parcial con el estudio de Mejía que determinó que los niños en edades de 3 y 8 años necesitaron mayor atención odontológica. ${ }^{8}$ Por el contrario, en nuestro estudio los niños de 8 a 12 años fueron atendidos con menor frecuencia con un porcentaje del $22 \%$ del total de la población. La caries dental afecta entre un $60 \%$ a $90 \%$ de los escolares de 3 a 12 años, por lo tanto, es importante crear una cultura donde los niños acudan desde edades tempranas a revisiones odontológicas lo que permitirá diagnósticos oportunos para tratar o prevenir lesiones cariosas y otras enfermedades bucodentales, siempre apoyado por sus padres o representantes legales.

Con respecto a la frecuencia de las actividades de prevención realizadas en esta investigación la de mayor frecuencia corresponde a los sellantes con un porcentaje del $22 \%$ y profilaxis con un $17 \%$ lo que concuerda con lo publicado por Milgrom. $\mathrm{P}^{10}$ donde las actividades de prevención se realizaron en edad temprana ya sea 
en dentición decidua o dentición permanente logrando disminuir los índices de CPOD en edad adulta. Se encontró coincidencia con el estudio realizado por Delgado, col $^{11}$ donde la frecuencia de sellantes fue mayor con $10.8 \%$ que las restauraciones atraumáticas con un $5.8 \%$. Se ha demostrado que la efectividad de los sellantes para eludir la caries va desde un $83 \%$ después de un año, hasta un $53 \%$ después de 15 años ${ }^{12}$ concluyendo que la aplicación de procedimientos preventivos es efectiva en el control y prevalencia de caries además de mejorar la higiene oral. Además se ha demostrado que la prevención es la mejor alternativa para eludir el desarrollo o el progreso de las enfermedades, por lo cual este estudio presento mayor cantidad de actividades preventivas en la población estudiada mediante enseñanzas de higiene oral y charlas educativas además de la aplicación de sellantes, flúor y profilaxis para reducir las enfermedades bucodentales a futuro.

De acuerdo a las actividades de restauración la de mayor frecuencia en este estudio fue la operatoria dental con un $22 \%$ resultados similares reporta el estudio de Fox y cols $^{13}$ donde se realizó una mayor cantidad de restauraciones atraumáticas que exodoncias en una comunidad. Se realizó mayor cantidad de operatorias en dientes deciduos y permanentes con la finalidad de devolver la funcionalidad, estética y fonación del paciente además de resultar un tratamiento menos invasivo que genera menor dolor y ansiedad que la extracción dental en los niños.

Según el grado de escolaridad obtuvimos que la mayor participación pertenece al Centro de Educación Básica Inicial Pitufos Del Valle con un $43 \%$, resultados que no concuerdan con el estudio aplicado en comunidades vulnerables limitadas al acceso de salud pública realizado por Mejía y cols ${ }^{8}$ donde la mayor participación fue en niños con grado de escolaridad Básica media. Se debe considerar que existió una mayor participación de los estudiantes de esta la Unidad Básica debido a que toda su población está compuesta por niños de 0 a 3 años y este proyecto intervino en el $100 \%$ de su población. Es importante trasmitir a los padres la importancia del cuidado dental de sus hijos y la responsabilidad que ellos tienen hasta que el niño adquiera las destrezas motoras para hacerlo por sí solo que por lo general no sucede después de los 7 u 8 años, donde entonces el adulto responsable puede seguir supervisando al menos el cepillado nocturno. Por lo cual demuestran la necesidad de programas de intervención para comunicar a las comunidades vulnerables la importancia de adquirir correctos hábitos de higiene oral desde la infancia temprana para reducir el riesgo de caries dental.

\section{Conclusiones}

Dentro de este estudio podemos destacar que el sexo masculino con un rango de edad de 0-3 años, tuvo mayor participación dentro de las actividades de prevención, además de acuerdo a la frecuencia de las actividades realizadas, tenemos que la colocación de sellantes fue la actividad de prevención que más se realizó, mientras que de las actividades de restauración las más frecuentes fueron las operatorias, finalmente según el grado de escolaridad quienes tuvieron mayor participación en este estudio fueron los niños de Inicial Básico pertenecientes al Centro de Educación Básica Pitufos Del Valle. Resaltando la necesidad de programas de intervención que tengan el objetivo de comunicar a las familias la importancia de obtener hábitos de higiene oral desde la edad temprana.

Este estudio cuenta con el apoyo de la Institución y fue autofinanciado.

Conflicto de intereses: Los autores del presente estudio manifiestan que no existe ningún conflicto de intereses en relación al tema de estudio.

\section{Referencias}

1 Hidalgo Rodríguez H, Conto Díaz F, Gaibor Duran A, Pincay Criollo J. Visión 2030 de la odontología en el Ecuador. Rev. Científica Dominio de las Ciencias. Disponible en: [Internet]. 2017 [citado 2020 abril 26]; 3(2): 788-809.

2 Contreras A. La promoción de la salud general y la salud oral: una estrategia conjunta. Rev Clín Periodoncia Implantol Rehabil Oral. [Internet].2016 [citado 2020 abril 26];9(2):193-202.

3 Cupé A, García C. Conocimientos de los padres sobre la salud bucal de niños preescolares: desarrollo y validación de un instrumento. Rev Estomatol Herediana. [Internet].2015 [citado 2020 abril 26];25(2):112-121.

4 Cubero A, Lorido I, González A, Ferrer M, ZapataM, Ambel J. Prevalencia de caries dental en escolares de educación infantil de una zona de salud con nivel socioeconómico bajo. Rev Pediatr Aten Primaria. 2019;21:e47-e59.

5 Sanabria D. Et al. Caries dental en menores en situación de pobreza, asistidos por una fundación en Paraguay. Rev Estomatol Herediana. [Internet].2016 [citado 2020 abril 26];26(2):70-7.

6 Ministerio de Salud Pública, "Programa Nacional y Normas de Estomatología" Junio, 2009.

7 Cabeza G, González F, Paredes C. Estado de salud oral en el Ecuador. Revista OACTIVA UC. 2016;1(3):65-70.

8 Mejía I, Gutiérrez D, Castro C, Rodríguez L. Salud bucodental para comunidades vulnerables y desplazadas de Popayán que viven en condiciones de pobreza. Rev. Cubana de Salud Pública. 2019;45(3). 
9 Ojeda R, Dávila K. Prevalencia de caries dental en niños de la clínica estomatológica de la Universidad señor de Sipán. Rev. Salud \& Vida Sipanense. 2017;4(2).

10 San Andrés Plua Nelly, Parra Cruz Addys, Espinosa Estrella Wilson, Santos Zambrano Thaináh. Salud bucodental de los escolares. Unidad Educativa Fiscal "Franklin Delano Roosevelt”. Rev San Gregorio. [Internet]. 2018 Oct [citado 2020 mayo 25]; 24(1): 90-97.

11 Delgado Angulo Elsa, Bernabé Ortiz Eduardo, Sánchez Borjas Pablo. Análisis de supervivencia de sellantes y restauraciones ART realizados por estudiantes de pregrado. Rev Estomatol Herediana. [Internet]. [citado 2020 mayo 25]; 15(2): 76-80. Disponible en: https://www.redalyc.org/articulo.oa?id=421539344004

12 Villarreal Rojas Azucena, Guerrero Ibarra Jorge, Yamamoto Nagano Adolfo, Barceló Santana Federico Humberto. Profundidad de curado de selladores de fosetas y fisuras utilizando luz emitida por diodos (LED) a diferentes distancias. Rev. Odont. Mex [Internet]. 2015 Jun [citado 2020 mayo 25]; 19(2): 76-80.

13 Fox, Mariela, Navas Perozo, Rita, Zambrano, Olga, Tratamiento de restauración atraumática (ART): una alternativa para el abordaje de comunidades vulnerables en estudios epidemiológicos. Ciencia Odontológica [Internet]. 2012;9(1):17-24. Disponible en: https://www.redalyc.org/articulo.oa?id=205225470004

Recibido: 11 de septiembre de 2020

Aceptado: 12 de noviembre de 2020 
